\title{
Study on Optimization of Cold Chain Logistics Distribution Based on Improved Particle Swarm Optimization Algorithm
}

\author{
Di Liang ${ }^{1, a}$, Shuang $\mathrm{Wu}^{1, \mathrm{~b}}$, Guizhi Sun ${ }^{1, \mathrm{c}}$ \\ ${ }^{1}$ School of Mechanical Engineering, Shenyang University, Shenyang, Liaoning Province, China \\ asydxld@163.com, 'c445391942@qq.com, bsungz1992@163.com
}

Keywords: PSO; cold chain logistics; optimization of distribution routing

\begin{abstract}
This paper made the lowest cost as the goal to establish the cold chain logistics optimization model according to the characteristics of cold chain logistics and distribution. The PSO was improved to make it suitable for logistics distribution issues. Finally, examples are verified that the improved PSO can solve the problem of cold chain logistics distribution better.
\end{abstract}

\section{Introduction}

Cold Chain Logistics is to ensure food quality in a refrigerated environment and reduce food consumption. It the logistics process of refrigerate that based on food frozen technology and refrigeration technology [1]. In recent years, the demand for cold chain products continues to grow as people's pursuit of high-quality and accelerated pace of life, it takes the cold chain logistics industry an enormous development space. In the cold chain logistics industry, perishable food production or distribution companies self-logistics of suppliers are coexist with third party logistics, the ratio of logistics quantity that the former accounts for about 80 percent, the latter is only 20\% [2], so our cold chain logistics is still in the early stages of development. In 1894 American Albert Scott Barr concept Lille (Albert Barrier) and the British OA Leddy Qi (0.A.Ruddich) proposed cold chain (Cold Chain) [3]. Compared with the development of foreign food cold chain logistics, domestic in the related research is still in its infancy.It had more representative that Liu Beilin put forward the food cold chain , he said that food cold chain was a systems engineering, perishable foods had been in low temperature in the production, storage, transportation, sales and consumption before all of the link environment to reduce loss of guaranteed quality of food [4]. Wang Lan introduced the concept of food cold chain, studied the problem of food cold chain development in China, and in-depth analyzed the causes of the problem [5].

The experts and scholars study the problems of cold chain logistics from different angles, but it is mostly for the theoretical study, planning methods involved calculation are less. This paper selects PSO to solve the problem of cold chain logistics and route optimization, the algorithm is simple and easy to implement. POS has superiority in solving optimization problems by example .

\section{The establishment of cold chain logistics distribution model}

Model assumption. In order to facilitate research, the paper needs to make model assumptions. Assuming the transport of goods is a single variety; Vehicles are freezing or frozen vehicles has of the same capacity, the same type and condition in transportation process; the maximum carrying capacity of per refrigerated transport vehicle is larger than the demand for a single customer, transport vehicles are in good capable of running and they can complete customers' transportation task one time; This model study only one way, it don't study the round-trip; Each customer demand for the goods, the distance between any two customers, and the distribution distance from center and the customer are known; Each customers must accept the services of distribution center, it can only accept a refrigerated vehicle's services; cargo needs to be saved under suitable conditions in the process of transportation and warehousing. 
Model establishment. In this paper, make the cost minimum as the target to build, objective function is as follows:

$$
\begin{aligned}
& \operatorname{Min} C=\omega_{1} \sum_{l=1}^{n} A(l)+\omega_{2} \sum_{i=0}^{m} \sum_{j=0}^{m} \sum_{l=1}^{n} X_{i j}^{l} B_{i j}^{l}+\omega_{3} \sum_{j=1}^{m} \sum_{l=1}^{n} Y_{j}^{l}\left(a_{1}\left(T_{j}^{l}-T_{j}\right)+a_{2} \frac{d_{j}}{V_{j}^{l}}\right) \\
& +\omega_{4} C_{1} \sum_{j=1}^{m} \sum_{l=1}^{n} Y_{j}^{l} Q_{j}^{l}\left(a_{3}\left(T_{j}^{l}-T_{j}\right)+a_{4} \frac{d_{j}}{V_{j}^{l}}\right)+\omega_{5} \sum_{j=1}^{m} c_{2} b W_{j}^{l}
\end{aligned}
$$

There are $m$ customers and $n$ Vehicles, $\omega$ represents the weight of the overhead costs, $A(l)$ represents the fixed cost of the vehicle, this formula represents their vehicles' fixed costs; $B_{i j}^{l}$ is vehicle transportation cost on the arc $(i, j), X_{i j}^{l}$ is the decision variable, $X_{i j}^{l}$ is equal to 1 when the vehicles pass the arc $(i, j)$, otherwise equal to 0 , this formula represents transport cost; $Y_{j}^{l}$ is the decision variable, it indicates whether service for customers, $a_{1}$ represents a unit energy consumption ratio during transport, $a_{2}$ represents the ratio of units energy consumption in the process of services for customers, $T_{1 j}^{l}$ represents the time from distribution center to customer, $T_{2 j}^{l}$ represents the time of customer service, this formula is energy consumption cost; $c_{1}$ represents the unit cost of goods, $Q_{j}^{l}$ represents remaining weight of the goods on vehicles when $l$ vehicle reach the customer, $a_{3}$ represents the proportion of units lost during transportation, $a_{4}$ represents unit loss ratio in the process of customer service, this formula is the cost of cargo loss; $c_{2}$ represents the punitive cost of goods unit damage amount, $b$ represents food spoilage rate, $W_{j}^{l}$ represents good amount of goods during transport, this formula is the punishment cost.

The formula must meet the following constraints:

$$
\begin{gathered}
\sum_{l=1}^{n} N_{l}=m \\
\sum_{l=1}^{n} X_{j}^{l}=1(j=1 \ldots . . m) \\
P_{l} \leq L ; \\
\sum_{j=1}^{m} X_{j}^{l} d_{j} \leq G_{k}(j=1 \ldots . . m)
\end{gathered}
$$

(2) ensure that the number of clients receiving services on all distribution paths is equal to the total number of customers; (3) ensure that each client must receive services of distribution center, and accept service of a refrigerated vehicle; (4) ensure that each vehicle's actual driving distance does not exceed the limit distance of each vehicle; (5) ensure that each customer demand sum on the distribution route does not exceed the vehicle's maximum load capacity.

\section{Improve the PSO}

PSO is the algorithm of continuous space, and distribution optimization problem is an integer programming problem, so it has to be modified accordingly, the specific process is as follows:

A. Make adjacent particle swarm divide into several overlapping adjacent sub-group;

B. Use the fitness function to evaluate all the particles;

C. Make the initial adaptation value as the history of individual optimal solution, find a local particle swarm optimal solutions and global optimal solution; 
D. Use suitable function as an evaluation criterion

E. For each particle, it will adapt to the current value of its historical best fitness particles compared to the value, if the current is better than the history, the current position vector is the best position particle;

F. Each particle of PSO is related to compare with the history of individual optimal values and global optimum, then decide whether update the calculation result;

G. Determine whether termination condition is satisfied, if it meet, transfer the next step, or re-initialized.

The flow chart is shown in Figure 1:

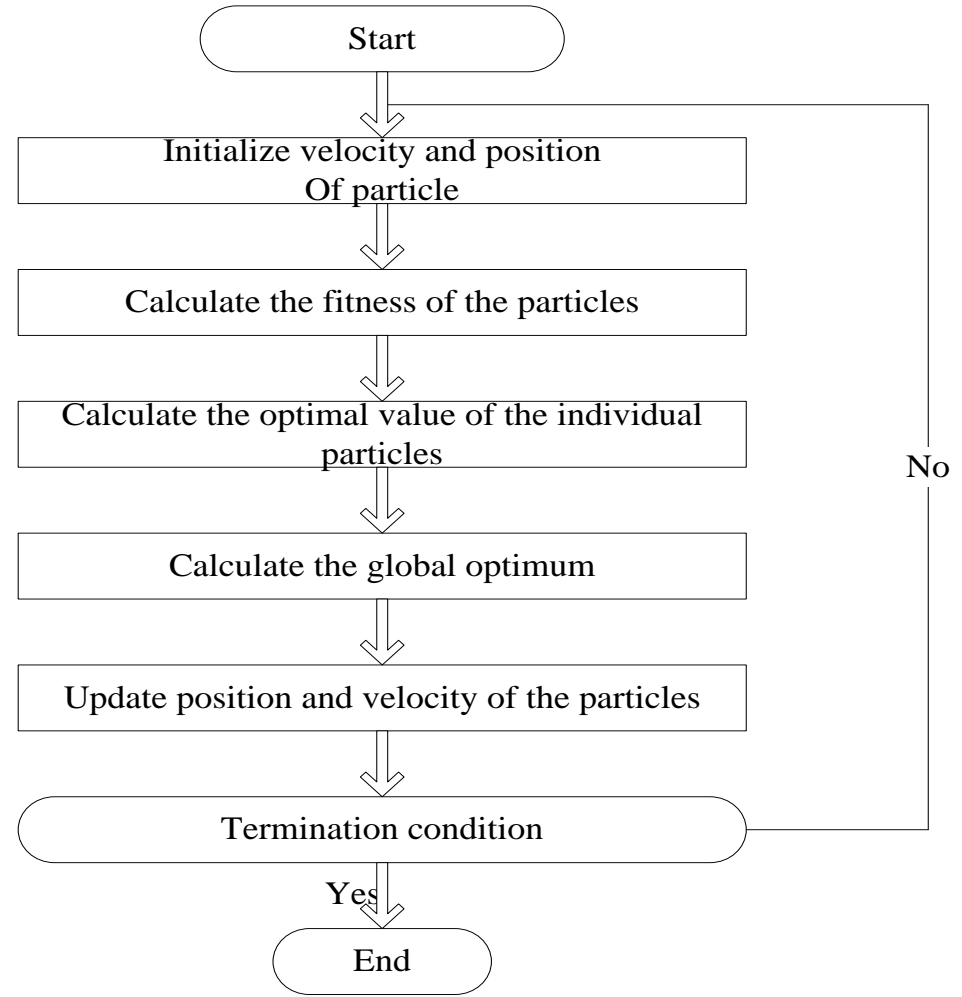

Fig. 1 PSO flowchart

\section{Example verification}

Distribution center has 10 cargo transport tasks, numbered 1,2,3,4,5,6,7,8,9,10. Distribution Center numbers 0. Distribution of goods is the seafood. During transport there are only one distribution center and 10 clients. All vehicles have the same model, same age, same condition.The level of driver's driving is same, the rate of logistics operators' loading and unloading is same The maximum carrying capacity of the vehicle is three tons. Fixed cost of the vehicle is 350 yuan / times. The unit cost of seafood is 20,000 yuan / ton. The per mile transport costs of refrigerated vehicles is 2.5 yuan / km the speed is $30 \mathrm{~km} / \mathrm{h}$. The shelf life of seafood is 60 hours at room temperature. The proportion of cargo damage is 0.0008 per unit time during transport, the proportion of cargo damage unit time can be calculated as 0.0012 per hour in the process of customer service. In the process of cargo loading and unloading, loading and unloading rate per of unit time is 1.4 tons / hour. Energy cost is 4 yuan / hour. The penalty factor is set to 0.0019 units / hour. To study conveniently, the right to the cost of re-argument is 1 .

The distance from distribution center to each customer, and the seafood demand of each customer are as shown in Table 1: 
Table 1 The distance from distribution center to each customer and the seafood demand

\begin{tabular}{|c|c|c|c|c|c|c|c|c|c|c|c|c|}
\hline Distribution sites & \multicolumn{11}{|c|}{ The distance to each distribution sites $/ \mathrm{km}$} & Demand/ton \\
\hline $\mathrm{M}_{\mathrm{ij}}$ & 0 & 1 & 2 & 3 & 4 & 5 & 6 & 7 & 8 & 9 & 10 & \\
\hline 0 & 0 & 12 & 23 & 24 & 15 & 26 & 16 & 19 & 25 & 16 & 20 & \\
\hline 1 & & 0 & 11 & 20 & 12 & 20 & 25 & 24 & 15 & 19 & 17 & 0.79 \\
\hline 2 & & & 0 & 23 & 23 & 19 & 18 & 13 & 16 & 26 & 18 & 0.71 \\
\hline 3 & & & & 0 & 14 & 21 & 24 & 19 & 16 & 17 & 25 & 1.08 \\
\hline 4 & & & & & 0 & 15 & 18 & 12 & 25 & 31 & 31 & 1.28 \\
\hline 5 & & & & & & 0 & 18 & 13 & 25 & 17 & 14 & 0.62 \\
\hline 6 & & & & & & & 0 & 15 & 11 & 22 & 30 & 0.91 \\
\hline 7 & & & & & & & & 0 & 21 & 27 & 15 & 1.17 \\
\hline 8 & & & & & & & & & 0 & 9 & 14 & 1.10 \\
\hline 9 & & & & & & & & & & 0 & 10 & 1.52 \\
\hline 10 & & & & & & & & & & & 0 & 1.40 \\
\hline
\end{tabular}

The maximum number of iterations is $T_{\max }=300$. The number of population particle is 200 . Achieve rapid searching with MATLAB7.0, the optimal value stabilizes when iterative times are about 80 times, the optimal distribution path are follow:

Vehicle 1 takes 710.21 yuan: $0-->4-->3$;

Vehicle 2 takes 785.03 yuan: $0-->1-->2->8$;

Vehicle 3 takes 822.38 yuan:0-->9-->10;

Vehicle 3 takes 833.11 yuan:0-->6-->7-->5;

The final optimization extreme is that:

Using PSO can find an optimum solution easily and effectively for cold-chain logistics distribution routing problem.

\section{Summary}

Firstly, this paper established the cold chain logistics optimization model that take the lowest-cost as the target based on the characteristics of cold chain logistics. It gave full consideration to the cold chain distribution process various fees and costs, included vehicle fixed costs, transportation costs, loss costs, energy consumption penalty cost. Secondly, improve the PSO, PSO was applicable to solve the problem of cold chain logistics after improvement. Finally, take a numerical example to verify, improved PSO can solve optimization problems and distribution chain logistics rightly and quickly.

\section{References}

[1] W. Yang, G.D. Li, Q. Zhang: Journal of Shaanxi University of Science\&Technology, Vol. 31(2013), p.30-34

[2] Golan E, Krissoff B, Kuchler F: Dead or Super Highway, Vol.28(2003), p.17-20

[3] Q. Wang: Sun Logistics, Vol.1(2004), p.20-21

[4] W.Z. Liu, M.Y. Sun: Logistics \& Material Handling, Vol. 15(2009), p.82-86

[5] L. Wang: International Conference on Management of Commerce and Government, Vol.8(2008,), p.18-21 